\title{
Student insight about the discipline of surgical technique on the formative process in the School of Medicine of UFRJ ${ }^{1}$
}

\author{
Victor Diniz Senna', Keren Cozer", Manoel Luiz Ferreira"', José Marcus Raso Eulálio"', Paulo Cesar \\ Silvalv, José Eduardo Ferreira Mansov, Alberto Schanaider ${ }^{v 1}$
}

\begin{abstract}
'Graduate student, Department of Surgery, School of Medicine, Universidade Federal do Rio de Janeiro (UFRJ), Brazil. Design of the study; acquisition, analysis and interpretation of data; statistical analysis, manuscript writing.

"Scientific initiation student, Grant from Institutional Program for Scientific Initiation (PIBIC), Na-tional Council of Technological and Scientific Development (CNPq), Rio de Janeiro-RJ, Brazil. Manu-script preparation.

I'PhD, Assistant Professor, Department of Surgery, School of Medicine, UFRJ. Rio de Janeiro-RJ, Bra-zil. Acquisition and interpretation of data.

IVPhD, Assistant Professor, Head, Department of Surgery, School of Medicine, UFRJ, Rio de Janeiro-RJ, Brazil. Acquisition and interpretation of data.

'PhD, Associate Professor, Head, Postgraduate Program in Surgical Sciences, Department of Surgery, School of Medicine, UFRJ, Rio de Janeiro-RJ, Brazil. Acquisition and interpretation of data.

${ }^{V 1}$ Full Professor, Head, Center of Experimental Surgical. Coordinator, Postgraduate Program in Surgical Sciences, Department of Surgery, School of Medicine, UFRJ, Rio de Janeiro-RJ, Brazil. Conception and design of the study, manuscript writing, critical revision.
\end{abstract}

\section{Abstract}

Purpose: To evaluate whether undergraduate students feel motivated to develop surgical skills and know their impression about the importance of having a surgical technique discipline in the curriculum of a medical school.

Methods: A prospective study including three classes in a row, from the 8th period $(n=265)$ evaluated the knowledge acquired at the Center of Experimental Surgery, Department of Surgery, School of Medicine of Federal University of Rio de Janeiro (UFRJ). The importance of the discipline for medical training as a way of encouragement to arouse and deepen the interest in surgical technique was emphasized. The questions were scored from 1 to 5 (worst to best grade).

Results: Concerning the importance of the discipline for medical training, $78 \%$ and $18 \%$ of the students assigned a score 5 and 4, respectively. Regarding the stimulus to improve their surgical technical skills, 40\% and 32\% attributed the score 5 and 4, respectively.

Conclusion: Undergraduate students from the Medical School of UFRJ effectively shared the understanding that the operative technique bases improve the formative process with significant impact, not only in the development of skills, but also to arouse vocations and stimulate new attitudes aimed to knowledge acquirement in the field of Surgery.

Key words: Education, Medical, Undergraduate. Surgery, Educational Measurement. 


\section{- Introduction}

The evaluation of the teaching-andlearning process in the context of basic training in Surgery, in the curriculum grid of a medical school comprises, traditionally, the measurement of acquired skills and competencies in simulation and operative practices scenarios. We observe a strong focus on motor aspects assessment, technical skills incorporated and collective performance. However, few studies have evaluated the perception of the undergraduate student on the impact of curriculum content in medical training and on motivation acquired for future professional practice.

The early insertion of students in a health public environment in order to practice less complex surgical procedures, as it happens in Brazilian medical courses, has the power to motivate them to develop skills and even to apply to Surgery Residence Programs afterwards. However, one major challenge is to establish the ideal moment for this content to be taught in the medical school curriculum, having in mind that it is necessary to integrate prior knowledge to the scientific basis needed for the execution of basic surgical procedures safely.

It is noteworthy that various models designed for student training depend on the infrastructure of the teaching institution, which comes to be, most of times a public health service, and on tutors trained to supervise student activities. It is clear that the applicability of learned concepts and skills will require solid domain of operative technique scientific basis.

The discussion regards the medical course curricular grade and highlights the lack of studies about the students' points of view in different surgical practice scenarios and their consequences in professional life. There is an almost consensual recommendation that undergraduate students should participate in health services, in early stages of their formation. However, they do not have the skills needed for a proper performance. This scenario often demotivates students about a future inclusion in surgical activities. Hence, the temporal dissociation between the discipline of surgical skills basis and the habitual use of this knowledge, in the appropriate environment (ambulatory or emergency services) deserves some reflection and should be reevaluated. In the current context, there is still a potential risk of causing iatrogenic harm to patients, if practical activities are carried out without qualified supervision or if there is misconduct duo to lack of knowledge in performing minor surgical procedures.

It is usual hat students engage in a self-endeavor to seek complementary surgical activities outside the university hospital, known as a hidden curriculum search, in order to improve their knowledge. On the other hand, this strategy may be tricky and unsafe, especially when there is no supervision or in the absence of a professional with proper qualifications to provide the mentoring.

Future professional practice in surgical specialties keeps a close relationship with the student experience during the course, including both the excellence of the discipline and the charisma of its teachers. In addition, it is known that an effective stimulus provided by content taught in an ideal way and at an ideal time is capable of arousing awareness of the importance of the absorbed knowledge. Thus, the experimental surgery discipline, as a training locus of fundamentals of operative technique plays an essential role in medical training.

This study aimed to evaluate the perception of undergraduate students of a medical undergraduate school about the Principles of Surgical Technique discipline in the medical course curriculum, estimating its role as a stimulating agent for the acquisition of new skills in this area of knowledge. 


\section{- Methods}

The discipline Principles of Surgical Technique is currently taught in the eighth semester (fourth year) of medical school. It takes place at the Center of Experimental Surgery of the Department of Surgery, School of Medicine, Federal University of Rio de Janeiro (UFRJ), where there are facilities and infrastructure suitable for achieving both research and teaching objectives.

The curricular content includes simulations with less complex surgical procedures in emergency scenarios with concepts of asepsis and antisepsis (scrub up, wear gloves and surgical gown properly), surgical instruments, local anesthesia, knots and surgical sutures techniques in artificial skin simulators.

Ten to twelve students are positioned in two separate operating rooms in the presence of two professors and one undergraduate monitor or postgraduate student, both from the Department of Surgery, in each room, with a maximum ratio of two students per tutor. Each student has eight hours of practical activity and, half of the time is designated for skill improvement and content review. Besides the practical classes in the operating room, the course provides theoretical material and online demonstration videos.

At the end of the term, students who had completed the eighth semester of the School of Medicine of UFRJ had the chance to evaluate the Principles of Surgical Technique discipline. The study included the academic periods of 2015/2 (July to December), 2016/1 (January to June) and 2016/2 (July to December) with 84, 86 and 98 students respectively. 265 of the total amount of 268 questionnaires were considered valid. Three of them were considered not valid, comprising one blank and two filled in mistakenly, having a different content from the one taught in the discipline.

The two items addressed in this study were:

A: Give a grade to the importance of the discipline in your medical education

B: How did the discipline motivate you to improve your skills in operative technique?

The tests were applied on the last day of the course. The items were scored on a scale ranging from 1 (worse concept) to 5 (best concept). The grade 3 means intermediate or indifferent results. The students were not asked to identify themselves on the questionnaires, in respect to the desire of anonymity.

\section{Results}

The resulting analysis from the 167 questionnaires properly answered, obtained from both classes is illustrated on Table 1 , and Figures 1 and 2.

Table 1 - Number of responses graded in a scale (score 1 a 5).

\begin{tabular}{lccccc}
\hline Score & 5 & 4 & 3 & 2 & 1 \\
Item A & 207 & 48 & 8 & 2 & 0 \\
\hline Item B & 106 & 85 & 45 & 21 & 8 \\
\hline
\end{tabular}

The maximum grade on the item $A$ was given by $78 \%$ of students, i.e. they considered the discipline very important for medical formation. If the score 4 is also considered, the average rises to $96 \%$ of responses in the higher stratum (very important or with relevance). Only $3 \%$ rated as regular relevance or were indifferent (score 3) and another 1\% graded 
with score 2 (low relevance). Nobody scored 1 (without relevance) on item A (Figure 1).

The average of the answers of three classes in a row, showed that $40 \%$ of the students felt motivated (score 5) and another $32 \%$ considered it as a relevant stimulus (score 4). $17 \%$ were indifferent and $11 \%$ showed low or no motivation to improve their skills in surgical technique (Figure 2).

$2015.2+2016.1+2016.2$

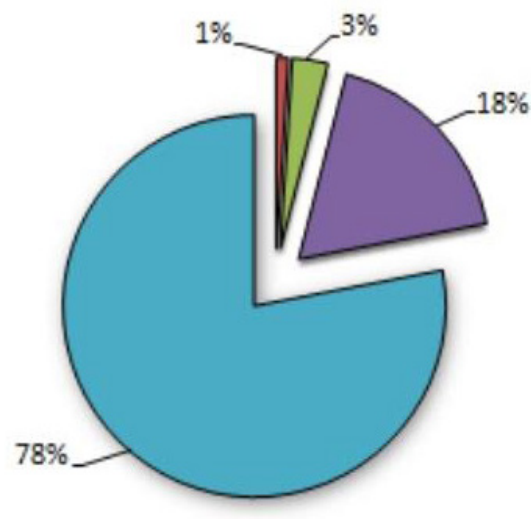

घ $(0 \%)$
$\square 2$
$\square 3$
$\square 4$
$\square 5$

Figure 1 - Importance of discipline for the medical formation. Analysis by class and average (2015/2, $2016 / 1$ and 2016/2). On the average, $96 \%$ of both group of students considered the discipline (Principles of Surgical Technique) relevant to medical education (scores 4 and 5).

\section{- Discussion}

What should be the goal of a Medical School? The answer is simple: to graduate good professionals. The Brazilian National Curriculum Guidelines of Medicine determine that the student must know how to perform medical and surgical medical procedures required to emergency care. However, it demands basic qualifications with not only a core curriculum but also with an appropriate infrastructure and teaching dedication, both essentials to improve an excellent medical

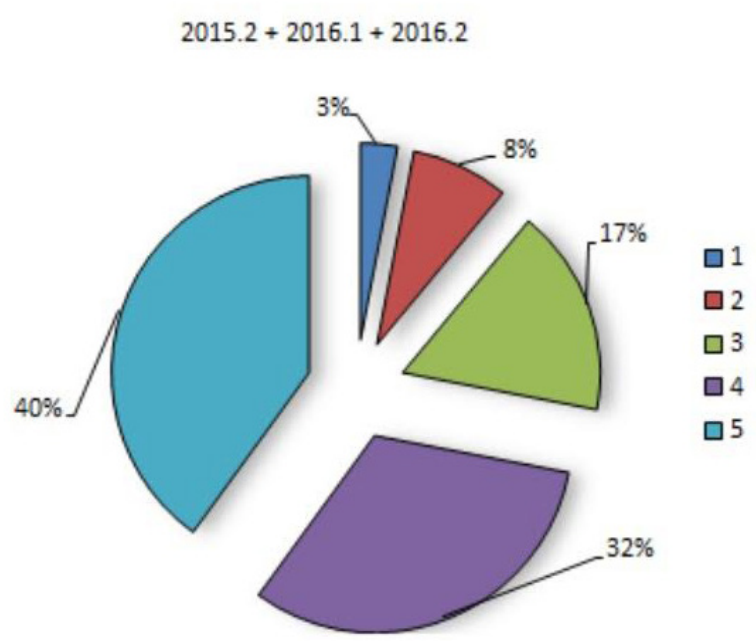

Figure 2 - Stimulus to improve surgical technique skills. Analysis by class and average (2015/2, $2016 / 1$ and 2016/2). Most of the students (72\%) felt motivated by the discipline classes (scores 4 and 5).

course. Among all disciplines, it is noteworthy that the old-style model of teaching surgery, regarding the traditional sequence of theoretical classes followed by demonstration and practice in crowded rooms does not fulfill the requirements for an adequate student preparation as far as it concerns to its future as a professional. We agreed with Thompson and cols opinion that skills training is a critical component of medical education, but when refers to surgery it is often neglected in standard curriculum ${ }^{1}$.

Several studies have shown students deficiencies due to the absence of indispensable opportunity of practicing under prepared tutor supervision. Most of times they follow the Aristoteles aphorism: "We are what we repeatedly do. Excellence, then, is not an act but a habit". However, in Medicine, not al-ways reproducing the surgical procedure in daily practice following the medical staff orientation means safe or quality assurance. It must be considered that a large part of practical learning occurs in public health ambulatory of 
Family Medicine or in later stages, in emergency rooms, where doctors, usually on duty, without any vocation for teaching, are raised to a tutor status. In such circumstances, formal learning objectives may conflict with staff routines, which can easily cause insecurity. Besides, another distress consists on the early insertion of students into primary health care performing minor surgical procedure without any scientific basis and with poor surgical knowledge. It should be considered that, frequently, students learn basic concepts while doing the procedure for the first time, exposing the patient to a greater risk of complications. Prior training in a controlled testing environment, like an exper-imental surgery lab, is essential to having good results in a future performance of surgical procedures in real patients. It is worth emphasizing that early insertion in health care does not mean that students must know how to suture or make a cricothyroidotomy, since well learned concepts concerning surgical scientific basis are necessary in first place.

In Brazil, most students do their internships in emergency services in the seventh or eighth semester of the medical course. We realize that the discipline of surgical technique can be more useful if taught concomitantly to practical activities in emergency scenarios, providing more efficient skill training. As we thought, Dehmer and cols have gotten to a similar conclusion ${ }^{2}$. They made a survey including 134 fourth-year medical students to analyze nine procedural skills taught during the course, including basic suturing, and showed that almost all students had performed most skills infrequently characterizing the existence of a gap between desired and actual level of competence in performing those procedures. They concluded that, at the end, students were both unable to perform those minor invasive procedures without assistance and unprepared to perform basic medical procedures on graduation.

We have structured an 8-hour surgical practice course linking demonstration, training, simulations (asepsis and gloving, identifying and using surgical instruments, antisepsis and performing local anesthesia, surgical knotting in artificial wound pad, suturing and removal of surgical sutures) and videos with online learning material. These learning tools emphasize, essentially, a hands-on practice with close teacher supervision. Clearly, in a group of students training surgical procedures, different motor skills and learning levels will appear. Therefore, the maximum ratio of $3: 1$ student/professor, sometimes with a help of a supervised monitor, is extremely beneficial for teaching-learning process. It is not only the repetition of the surgical procedures, but also a regular basis training without technical mistakes that bring up the most relevant principles for skills development. The vis-à-vis classes with a small ratio approach help the student to strengthen key aspects of the scientific knowledge, since theoretical components are introduced together with the practical activity and because of the opportunity to enlighten personal doubts, dealing with self-limitations. The students vividly recalled incidents when they were allowed to perform tasks themselves making it a great opportunity to reinforce the principles of the surgical technique.

A small number of manuscripts have pointed out the students' perceptions about the importance of the discipline of surgical technique for the future professional activity and the motivation for further studies in this field of knowledge ${ }^{3}$. Our program enable a student-centered teaching sustained in learning achievements of basic surgical skills and this point of view, considering the 265 valid questionnaires evaluated. To analyze the genuine impact of learning achievements value of an undergraduate surgical curriculum we asked two questions: "Assign a score to the importance of discipline to your medical formation" and "How do you evaluate the stimulus (of the discipline) to deepen your skills in operative technique?" Overall student ratings were very high for the first question, 
showing that almost all students (96\%) endorsed the statement. A positive assessment was also found for the second question. Students expressed a strong positive feedback directing their attention to improve skills in basic surgical technique. As such discipline often does not encourage students to reinforce their knowledge in this area, this should be seen as a remarkable fact.

Good standards in learning process may influence the career choice. Ek and cols, in a survey with final year medical students, found out a rate near $61 \%$ of students who declared themselves interested in surgical training, comparing to $72 \%$ obtained in our own survey ${ }^{4}$. Obviously, several factors influence such results including the quality of the discipline and the mentors' engagement ${ }^{5}$. We also agree that a well-structured discipline in the curriculum supports and encourages students towards a surgical career. It is not necessary to have a sophisticated infrastructure with computed based-video training to further improvements in skill development or retention ${ }^{6}$. The enthusiasm with the discipline goes beyond. Likewise, start cognitive motor learning for surgical skill in the operating room is not ethically accepta-ble and far from the ideal environment ${ }^{7}$. Positive experiences during the surgical disciplines surely have been associated with an increased interest in surgical careers but, above all, a good perception bout the discipline, having almost all our students positively endorsing the course, still depends on the teacher's role and its behavior.

\section{- Conclusion}

Undergraduate students from the Medical School of UFRJ effectively shared the understanding that the operative technique bases improve the formative process with significant impact, not only in the development of skills, but also in arousing vocations and stimulating new attitudes, aimed to knowledge acquirement in the context of Surgery.

\section{References}

1. Thompson L, Exline M, Leung CG, Way DP, Clinchot D, Bahner DP, Khandelwal S. A clinical pro-cedures curriculum for undergraduate medical students: the eightyear history of a third-year im-mersive experience. Med Educ Online. 2016 May 23;21:29486. doi: 10.3402/meo.v21.29486. eCol-lection 2016.

2. Dehmer JJ, Amos KD, Farrell TM, Meyer AA, Newton WP, Meyers MO. Competence and confi-dence with basic procedural skills: the experience and opinions of fourth-year medical students at a single institution. Acad Med 2013;88:682-7. doi: 10.1097/ ACM.0b013e31828b0007.

3. Liebert CA, Mazer L, Bereknyei Merrell S, Lin DT, Lau JN. Student perceptions of a simulation-based flipped classroom for the surgery clerkship: A mixed-methods study. Surgery. 2016 Sep;160(3):591-8. doi: 10.1016/j.surg.2016.03.034.

4. Nousiainen $M$, Brydges $R$, Backstein D, Dubrowski A. Comparison of expert instruction and com-puter-based video training in teaching fundamental surgical skills to medical students. Surgery. 2008 Apr;143(4):539-44. doi: 10.1016/j. surg.2007.10.022.

5. Ek EW, Ek ET, Mackay SD. Undergraduate experience of surgical teaching and its influence on career choice. ANZ J Surg. 2005 Aug;75(8):713-8. doi: 10.1111/j.14452197.2005.03500.x.

6. Zundel S, Meder A, Zipfel S, HerrmannWerner $A$. The surgical experience of current non-surgeons gained at medical school: a survey analysis with implications for teaching today's students. BMC Med Educ. 2015 Oct 27;15:187. doi: 10.1186/ s12909-015-0466-3.

7. Wong JA, Matsumoto ED. Primer: cognitive motor learning for teaching surgical skillhow are sur-gical skills taught and assessed? Nat Clin Pract Urol. 2008 Jan;5(1):47-54. doi: $10.1038 /$ ncpuro0991. 


\section{Correspondence:}

Alberto Schanaider

Avenida Carlos Chagas Filho, 373/BI J, 2 ㅇ andar

22461-200 Rio de Janeiro - RJ Brasil

Tel.: (55 21)3938-6549

albertoscha@gmail.com

Conflict of interest: none

Financial sources: CNPq and FAPERJ
Received: Sep 13, 2016

Review: Nov 16, 2016

Accepted: Dez 12, 2016

${ }^{1}$ Research performed at Center of Experimental Surgery, Department of Surgery, School of Medicine, Universidade Federal do Rio de Janeiro, Brazil. 